Research Paper

\title{
A Synergistic Therapeutic Scheme for Hyperglycemia and Nephrotic Disorders in Diabetes
}

\author{
Qingyi $\mathrm{He}^{2}$, Xing Zhang 3 , Baosan $\mathrm{Han}^{4}$, Jianzhong $\mathrm{Xu}^{2}$, Kanglai Tang ${ }^{2}$, Zhiren $\mathrm{Fu}^{1}$, Hao Yin ${ }^{1,5} \llbracket$ \\ 1. Department of Surgery, Organ Transplant Center, Shanghai Changzheng Hospital, Shanghai Second Military Medical University, \\ Shanghai, China 200003. \\ 2. Department of Orthopedic Surgery, Southwest Hospital, Third Military Medical University, Chongqing, China 400038. \\ 3. School of Engineering and Applied Sciences, Harvard University, Cambridge, MA 02138 USA. \\ 4. Department of Surgery, Shanghai Xinhua Hospital, Shanghai, China 200003. \\ 5. Department of surgery, University of Chicago, Chicago, IL 60637 USA.
}

$\square$ Corresponding author: Hao Yin, MD. Department of Surgery, Organ Transplant Center, Shanghai Changzheng Hospital, Second Military Medical University, 415 Fengyang Road, Shanghai, China 200003. E-mail: haoyin.sh@gmail.com Fax: 86-21-81885754 Tel: 86-21-81886999.

( ) Ivyspring International Publisher. This is an open-access article distributed under the terms of the Creative Commons License (http://creativecommons.org/ licenses/by-nc-nd/3.0/). Reproduction is permitted for personal, noncommercial use, provided that the article is in whole, unmodified, and properly cited.

Received: 2013.10.09; Accepted: 2014.01.28; Published: 2014.03.05

\begin{abstract}
We previously demonstrated that the utilization of an electrospun scaffold could boost functional outputs of transplanted islets. In this study, we aim to develop a drug-eluting scaffold with a payload of pioglitazone to simultaneously rein in hyperglycemia and recoup lost renal functions in diabetic mice that underwent islet transplantation. The in vivo proliferation of islets was measured by a non-invasive bio-imaging technology whereas the blood insulin, blood glucose and renal proteins were assayed. The local stimulation of transplanted islets by pioglitazone saw an accelerated in vivo proliferation without apoptosis caused by the drug-eluting scaffold. In addition, pioglitazone contributed to an increased secretion of insulin and C-peptide 2, giving rise to an accelerated rein-in of hyperglycemia and enhanced tolerance of sudden oral glucose challenge. Moreover, the accelerated decrease of blood creatinine, urine creatinine and blood urea nitrogen suggested that pioglitazone contributed to the recovery of renal functions compromised by diabetes. Our bioengineering strategy effectively ameliorated hyperglycemia and associated nephrotic disorders, and shed a new light on an engineering approach to combat diabetes.
\end{abstract}

Key words: pioglitazone; diabetes; islet transplantation; electrospinning; scaffolds.

\section{Introduction}

Diabetes and its associated diseases like renal artery stenosis have been leading cause of deaths around the world for decades. In spite of significant progress in diabetic research in the last decades, islet transplantation has remained a popular clinical solution for diabetic patients to restore insulin secretion and to alleviate the hyperglycemia. Recent research suggested that insulin-secreting pancreatic $\beta$-cells could increase their proliferation in response to an enhanced demand for insulin and physiological injuries.[1-4] This discovery shed a new light on the therapeutic approach for diabetic patients by stimu- lating $\beta$-cells in islets to control the blood glucose level. Unfortunately, clinical observations only presented a very limited number of supportive case reports with the majority of patients demonstrating insufficient capability to secret adequate insulin for blood glucose hemostasis.[5-9]

Tissue engineering scientists have extensively explored how to employ biomaterials to engineer functional tissues to recoup lost or compromised functions.[10-15] However, unfortunately, efforts dedicated to diabetes remains scant. This deficiency led us to focus an investigative spotlight on the use of 
tissue engineering scheme to improve existing treatments of diabetes. In current clinical practice, harvested islets are freely injected into patients' intrakidney capsule. It has been noted that transplanted islets suffered a poor survival rate due to a variety of reasons like local inflammation, effectively requiring to a large pool of islets at the time of transplantation. These limitations prompted us to employ an electrospun scaffold to boost the functional output of islet transplantation.[16] We demonstrated that the use of an electrospun scaffold helped to accelerate the growth of transplanted islets, increased the level of blood insulin, and improved the management of hyperglycemia. Pioglitazone, a member of the thiazolidinedione drug family, is clinically used to enhance the tissue sensitivity to insulin in diabetic patient alone or with other drugs.[17] Our previous research demonstrated that pioglitazone, either alone or with other anti-diabetic drugs, was effective in alleviating hyperglycemia by stimulating pancreatic $\beta$-cells for increased secretion of insulin.[18] Unfortunately, pioglitazone causes a potent immuno-suppression effect in clinical treatment, rendering diabetic patients venerable for various complications.

In light of these research findings and clinical challenges, we aim to functionalize our electrospun scaffold so that it is capable of locally delivering pioglitazone to transplanted islets. This local delivery would stimulate the production of insulin and thus lead to a better control of blood glucose in diabetic patients whereas renal functions could be simultaneously restored. Meanwhile, this targeted delivery would dramatically decrease the required dosage of pioglitazone and minimize adverse side effects associated with pioglitazone. The success of this strategy would give physicians an alternative therapeutic solution to cope with the complex situation in diabetic patients.

\section{Materials and Methods}

\section{The scaffold fabrication and the morphological characterization}

The scaffolds were fabricated as reported previously.[16] To fabricate the non-eluting scaffold (NES), PGC (Advanced Inventory Management, Mokena, IL) and PCL (Absorbable Polymers, Birmingham, AL) were dissolved (weight ratio of 1:3) in 1,1,1,3,3,3-Hexafluoro-2-propanol (HFP) (Sigma Aldrich, St. Louis, $\mathrm{MO}$ ) to reach a total concentration of $12 \%(\mathrm{w} / \mathrm{v}) .0 .5 \mathrm{~mL}$ of the solution was electrospun through a 27 gauge blunt needle to a collection board distanced at $25 \mathrm{~cm}$ from the needle tip at $30 \mathrm{kV}$ voltage. The feeding rate was $3 \mathrm{ml} / \mathrm{hr}$. The NES scaffold was desiccated in vacuum for $24 \mathrm{hr}$ prior to subse- quent analyses. For drug-eluting scaffold (DES), pioglitazone $(12 \mathrm{mg} / \mathrm{ml})$ were added to the polymer solution and incubated at room temperature until it was completely dissolved. The pioglitazone-polymer solution was then electrospun under the same conditions as NES scaffolds. Scanning electron microscopy (SEM) was used to study the fiber morphology in respective scaffolds.

\section{The creation of MIP-luc transgenic mice}

MIP-luc transgenic mice (C57BL/ 6 background) that were able to express the firefly luciferase due to transgene containing an MIP promoter fragment were generated as reported previously.[16, 19] Each C57BL/ 6 mouse received one dose of streptozotocin (STZ) $(50 \mathrm{mg} / \mathrm{kg}$ ) (Sigma Chemical, St. Louis, MO) by i.p. injection every two days for a total of five doses. Diabetes was confirmed when the blood glucose concentration exceeded $500 \mathrm{mg} / \mathrm{dl}$ for at least two consecutive days in the absence of fasting.

\section{The in vitro characterizations of electrospun scaffolds}

Respective scaffolds were incubated in cell media (2ml aliquots) at $37^{\circ} \mathrm{C}$ and $5 \% \mathrm{CO}_{2}$ for up to 4 days and the in vitro kinetics of drug release was measured by a HPLC system equipped with a Symmetry $\mathrm{C}_{18}$ column (Waters Corporation) per manufacturer's protocol. Desiccated circular DES and NES scaffolds ( $D=6.5 \mathrm{~mm}$ ) were plated into a 96-well tissue culture plate (TCP) and incubated with $70 \%$ ethanol for $15 \mathrm{~min}$ followed by a thorough rinse of sterile PBS. Syngeneic islets from MIP-luc transgenic C57BL/6 mice were harvested by an intra-ductal collagenase digestion (Roche, Indianapolis, IN) and purification by Ficoll gradient centrifugation (Sigma Aldrich, St. Louis, MO) as previously described.[20, 21] Freshly collected islets were seeded (100 islet equivalents/scaffold) on the scaffold or directly on the bottom surface of TCP in islet growth media, and incubated for two days at $37^{\circ} \mathrm{C}$ with $5 \% \mathrm{CO}_{2}$. The viability of the islet population was measured by the tetrazolium compound [3-(4,5-dimethylthiazol-2-yl)-5 -(3-carboxymethoxyphenyl)-2-(4-sulfophenyl)-2H-tetr azolium,inner salt] (MTS) (Promega Corporation, Madison, WI) per the manufacturer's protocol. Apoptosis was measured using a Caspase-Glo® 3/7 assay kit per the manufacturer's protocols (Promega, Madison, WI, USA).

\section{The transplantation and proliferation of islets}

DES and NES were fabricated as stated above, sterilized in $70 \%$ ethanol and washed in sterile PBS. Islets from MIP-luc mice were transplanted with either DES or NES (100 islet equivalents/scaffold) under the kidney capsule while the diabetic mice were 
anesthetized with vaporized isoflurance following the established protocol.[16] All mice were euthanized 8 weeks after the transplantation. All experiments involving animals was reviewed and approved by Shanghai Changzheng Hospital to ensure the welfare of laboratory animals. The proliferation of transplanted islets was measured by the Xenogen IVIS 200 imaging system (Xenogen, Alameda, CA) at select time points after the transplantation as previously described.[16, 19] Briefly, after being fasted for $4 \mathrm{hr}$, the mice were anesthetized with vaporized isofluorane and positioned on the side for the initial image acquisition. Thereafter, each mouse received 15 $\mathrm{mg} / \mathrm{ml}$ D-luciferin in sterile PBS $(150 \mathrm{mg} / \mathrm{kg})$ by i.p. injection. The bioluminescent image was capture at 14 min after the injection. Image processing and bioluminescent measurement were performed using the Living Image Software (Xenogen).

\section{Immuno-staining of insulin-secreting cells after transplantation}

After the euthanasia, the kidney from each mouse containing transplanted islets were harvested and fixated in $10 \%$ formalin at $4^{\circ} \mathrm{C}$ for at least $24 \mathrm{hr}$. Then each sample was embedded in paraffin, sliced in $4 \mu \mathrm{m}$ thick and collected on charged glass slides. Anti-insulin antibody (Abcam, Cambridge, MA, USA) was used to stain the insulin-secreting cell in each sample.

\section{Functional output of transplanted islets}

A blood sample from each mouse was collected from the tail vein before the transplantation and then every two weeks until the euthanasia. No mice underwent fasting before the blood collection. The concentrations of C-peptide 2 and insulin were measured by a rat/mouse C-peptide 2 ELISA kit (Millipore, Bellirica, MA) and a rat insulin ELISA kit (Crystal Chem Inc., Chicago, IL), respectively, per manufacturers' protocols.[16] The blood glucose was measured with OneTouch Ultra glucometer (Lifescan, Johnson\&Johnson, Milpitas, CA). The oral glucoe tolerance test (OGTT) was performed on week 8 after the surgery. After the mice had been fasted for $16 \mathrm{hr}$, each mouse received glucose solution prepared with sterile water $(100 \mathrm{mg} / \mathrm{ml})$ (Sigma Aldrich) by oral gavage (2 $\mathrm{g} / \mathrm{kg}$ ). Blood glucose levels were measured using blood samples collected before the oral gavage ( $\min 0$ ) and at 30,60 and $120 \mathrm{~min}$ after the gavage. The serum concentration of pioglitazone was measured at week 0,4 and 8 following established protocols.[22, 23] The Immunoassays \& Multiplex Kits (EMD Millipore, Billerica, MA) were used to measure plasma cytokines, including monocyte chemoattractant protein-1
(MCP-1), interlukin-6 (IL-6) and interferon gamma $(\mathrm{IFN \gamma})$, per the manufacturer's protocol at week 0,4 and 8.

\section{Renal function recovery after the isle transplantation}

Blood and urine samples were collected immediately before the transplantation and then every two weeks after the surgery until the euthanasia. Blood creatinine (Abcam, Cambridge, MA), blood urea nitrogen (Bio Scientific Corp, Austin, TX), urine creatinine (Abcam) and urine albumin (Abnova, Walnut, $\mathrm{CA})$, were assayed following manufacturers' protocols as reported before.

\section{Data Analysis}

Images were processed with ImageJ. Student t-test with a Tukey test was used to analyze all data $(a=0.05)$. All results were presented as mean \pm standard deviation.

\section{Results}

\section{The characterizations of the DES scaffold}

The SEM micrographs showed no marked difference of fiber morphology between DES and NES groups (Figure 1). Our previous research demonstrated that the use of an electrospun PCL/PGC composite scaffold could lead to an improved management of blood glucose in diabetic mice due to the increased insulin from transplanted islets.[16] Those results suggested that the PCL/PGC scaffold provided a favorable microenvironment for islets to survive and proliferate without introducing cytotoxicity into the host. The lack of fiber morphology between DES and NES suggested that the enhanced functional output should be attributed to the therapeutic effects of pioglitazone, rather than structural cues from the scaffolds.

The in vitro kinetic study showed that pioglitazone saw a sustained release from DES for up to 4 days (Figure 2A). By day 4 , the accumulative release of pioglitazone reached $96.00 \pm 24.44 \%$ and saw no increase beyond day 4 . No pioglitazone was detected in the NES group (negative control). In the DES scaffold group, the serum concentration of pioglitazone reached $0.89 \pm 0.16 \mu \mathrm{g} / \mathrm{ml}$ at week 4 and dropped to $0.14 \pm 0.12 \mu \mathrm{g} / \mathrm{ml}$ at week 8 (Figure 2B). No pioglitazone were detected in the NES scaffold group. Neither MTS assay nor the apoptosis assay showed a difference among the NES, DES and TCP groups, suggesting that scaffolding materials and pioglitazone did not cause a significant apoptosis or jeopardized the viability of islets (Figure 2C and 2D). 


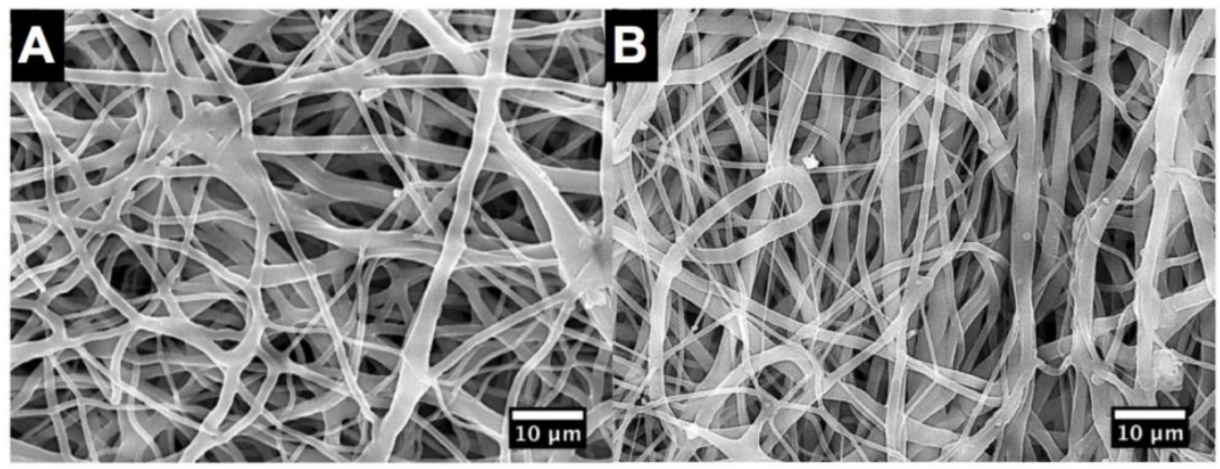

Figure I. The SEM micrographs of scaffolds. (A) non-eluting scaffolds (NES); (B) drug-eluting scaffolds (DES). No morphological difference was observed between NES and DES scaffolds. This result suggested that the difference of biomedical functional outputs was attributed to pioglitazone.

A

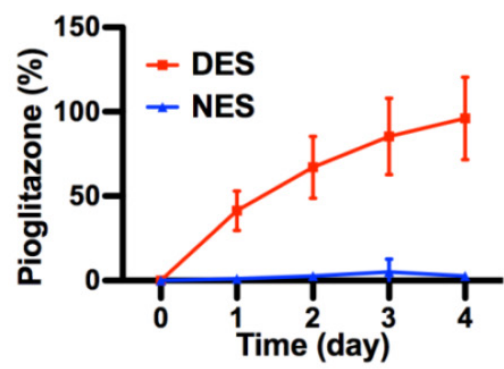

C

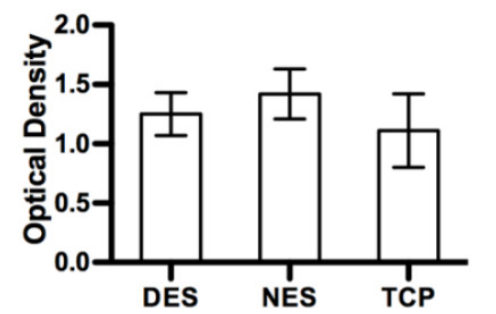

B

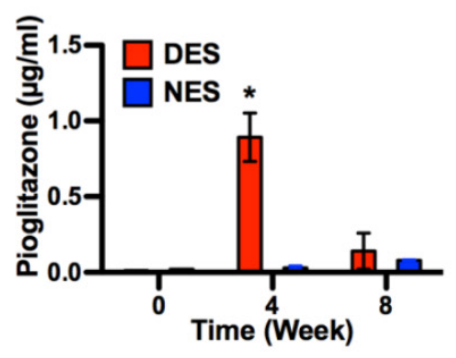

D

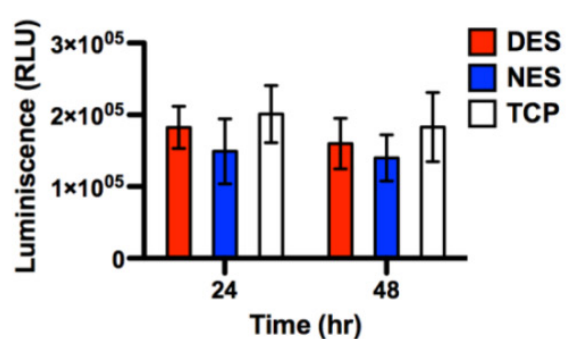

Figure 2. The characterizations of DES scaffolds. (A) The in vitro release of pioglitazone $(n=3)$. (B) The concentration of pioglitazone in serum $(n=8$ in $D E S$ group; $n=7$ in NES group). (C) The viability of islets cultured on DES, NES and tissue culture plates (TCP) $(n=3)$; (D) The apoptosis in islets cultured on DES, NES and TCP $(n=3)$. The DES scaffold could readily release pioglitazone for a limited time and exhibited no cytotoxic effects on islets.

\section{The in vivo proliferation of islet transplants}

The quantification of bioluminescent signal strength showed that the proliferation of islets accelerated in the presence of pioglitazone (Figure 3). The islet population from the DES group outgrew its peer from the NES group as early as week 4 and sustained this proliferative advantage through week 8 . The bioluminescent signal strength of the DES group was $43.59 \%$ and $34.93 \%$ larger than that of the NES group on week 4 and 8 , respectively. This sustained proliferation spoke to the fact that pioglitazone accelerated the growth of transplanted islets without provoking adversary effects like significant apoptosis.

\section{The functional outputs of transplanted islets}

The immunostaining demonstrated that pockets of viable insulin-secreting cells were under the in- trakidney capsule by the end of week 8 , evidencing that transplanted islets survived and reestablished their most critical function, that is, the insulin secretion (Figure 4). At week 4 after the surgery, the C-peptide 2 concentrations from the DES and NES groups were $45.81 \pm 6.01 \mathrm{pM}$ and $30.33 \pm 6.23 \mathrm{pM}$, respectively. Correspondingly, the insulin concentrations from the DES and NES groups were $239.23 \pm 30.11 \mathrm{pg} / \mathrm{ml}$ and $152.11 \pm 32.91 \mathrm{pg} / \mathrm{ml}$, respectively. Both C-peptide 2 and insulin showed a significant increase in the DES group at week 4 and this increase was observed through week 8 (Figure 5). Thanks to the increased insulin production in the DES group, the blood glucose level in the DES group returned to the physiological range $(<300 \mathrm{mg} / \mathrm{dl})$ on week $3(257.56 \pm 40.21 \mathrm{mg} / \mathrm{dl})$ and remained through week $8(213.45 \pm 27.45 \mathrm{mg} / \mathrm{dl})$. In contrast, the blood glucose level in the NES group remained abnormally 
high at week $4(362.11 \pm 49.45 \mathrm{mg} / \mathrm{dl})$ and only entered the physiological range on week 8 $(273.45 \pm 35.67 \mathrm{mg} / \mathrm{dl})$ (Figure 6A). The OGTT assay demonstrated that pioglitazone stimulated islets unleashed an enhanced tolerance to glucose challenge (Figure 6B). At $30 \mathrm{~min}$ after the glucose challenge, mice from the DES group showed a blood glucose concentration of $317.22 \pm 49.99 \mathrm{mg} / \mathrm{dl}$ whereas those from the NES group of $421.59 \pm 41.34 \mathrm{mg} / \mathrm{dl}$. Moreover, the glucose level in the DES group returned to physi-

A

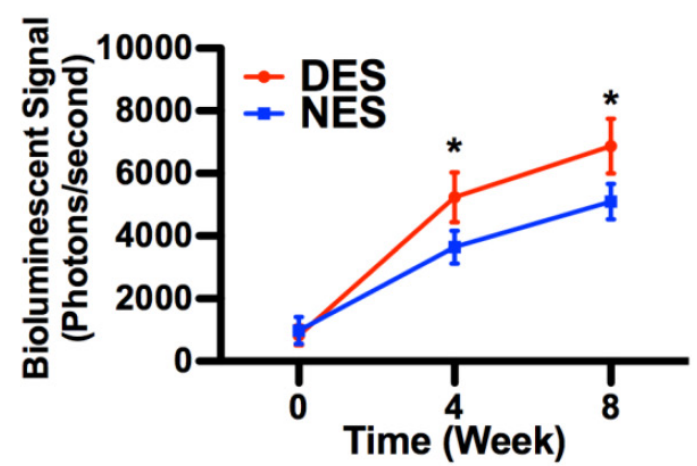

ological level by $60 \mathrm{~min}$ after the challenge $(227.56 \pm 40.21 \mathrm{mg} / \mathrm{dl})$ whereas the glucose level in the NES group remained abnormally high $(338.92 \pm 32.11 \mathrm{mg} / \mathrm{dl})$ by $120 \mathrm{~min}$. These results demonstrated that transplanted islets successfully survived and recapitulated their functions. Moreover, the stimulation of pioglitazone further enhanced the secretion of insulin and thus yielded an accelerated elimination of hyperglycemia compared to non-stimulated subjects.

B

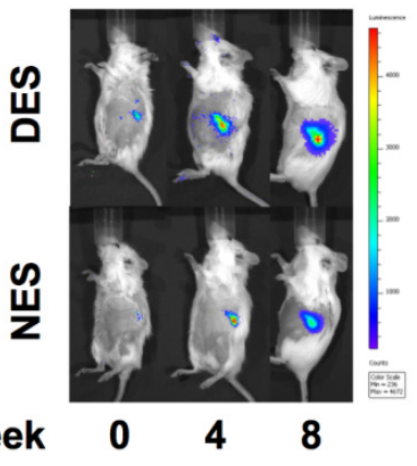

Figure 3. The in vivo proliferation of islets. (A) The strength of bioluminescent signals; (B) Representative bioluminescent images. The islets in the DES group outgrew those in the NES group ever since week 4 and maintained the lead through week 8 . This result suggested that pioglitazone caused a late yet long-term therapeutic effect rather than an immediate one. A star sign indicates a statistical difference between the groups ( $n=8$ in the DES group; $n=7$ in the NES group).
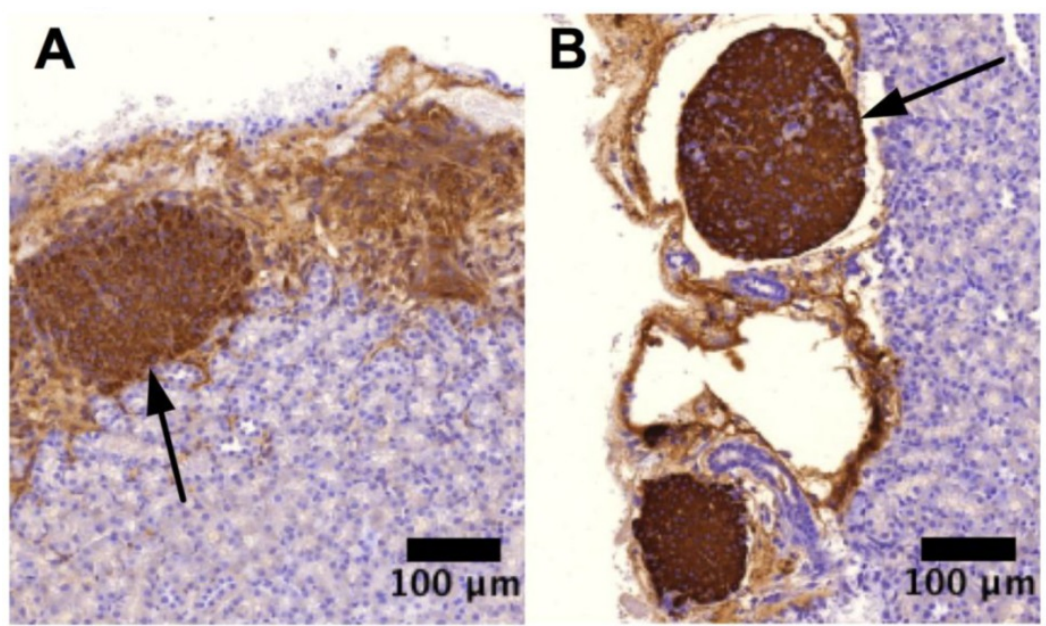

Figure 4. Insulin-secreting cells. (A) islet transplant from NES group; (B) islet transplant from DES group. The immuno-staining showed that islet transplants in both groups possessed their insulin-secreting capability. Arrows indicate the islet transplant in respective images.
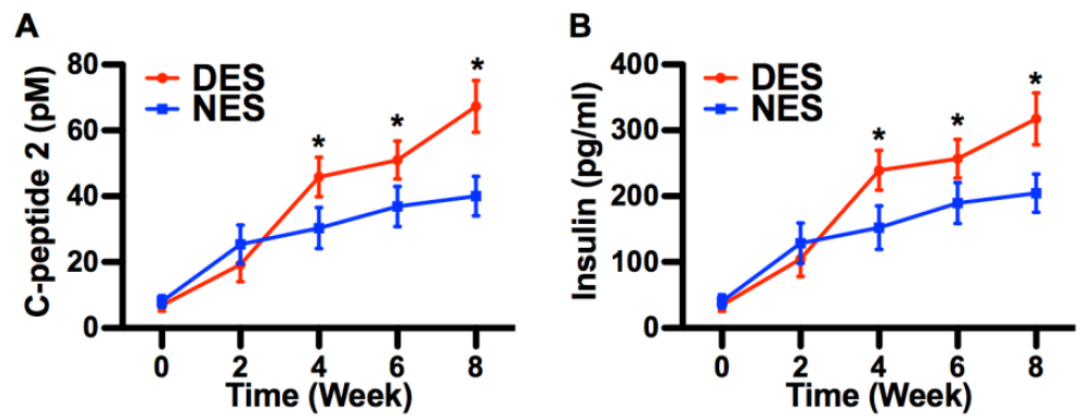

Figure 5. The insulin production by transplanted islets. (A) C-peptide 2; (B) Blood insulin. Both C-peptide 2 and insulin concentrations were higher in the DES group since week 4 . These results suggested that pioglitazone contributed to the increase of insulin secretion. A star sign indicates a statistical difference between the groups ( $n=8$ in the DES group; $n=7$ in the NES group). 

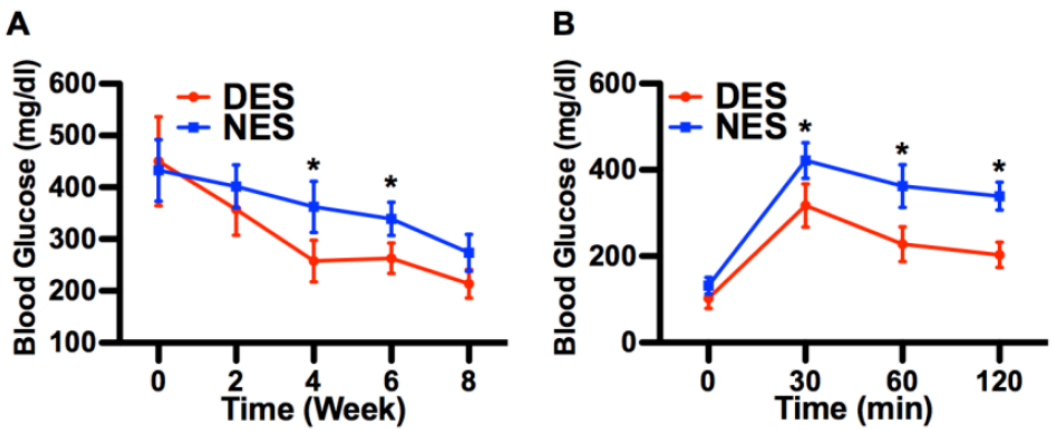

Figure 6. Hyperglycemia alleviation. (A) Blood glucose concentrations; (B) OGTT. The decrease of blood glucose concentration in the DES group outpaced that in the NES group. A marked difference emerged since week 4 . Consequently, mice in the DES group demonstrated a stronger tolerance of sudden glucose spike. These results suggested that pioglitazone contributed to the management of blood glucose. A star sign indicates a statistical difference between the groups ( $n=8$ in the DES group; $n=7$ in the NES group).

\section{The recovery of renal functions}

The concentrations of blood creatinine, urine albumin, blood urea nitrogen and urine creatinine are routinely used to assess the renal functions in diabetic subjects.[16] Following the islet transplantation, concentrations of all four proteins in both DES and NES group saw a steady decrease through week 8 (Figure $7)$. It is noteworthy that blood creatine, blood urea nitrogen and urine creatine in the DES group demonstrated an accelerated decrease compared to those in the NES group. At week 2, the concentrations of blood creatinine in the DES and NES groups were $1.42 \pm 0.27$ $\mathrm{mg} / \mathrm{dl}$ and $1.98 \pm 0.21 \mathrm{mg} / \mathrm{dl}$, respectively, whereas the concentrations of urine creatinine were $155 \pm 18 \mathrm{mg} / \mathrm{dl}$ and $211 \pm 24 \mathrm{mg} / \mathrm{dl}$, respectively. Similarly, the concentrations of blood urea nitrogen in the DES and NES groups were $48.99 \pm 12.11 \mathrm{mg} / \mathrm{dl}$ and $78.31 \pm 11.34$ $\mathrm{mg} / \mathrm{dl}$, respectively, at week 2 . However, the accelerated decrease of blood and urine creatinine in the DES group was lost by week 6 while that of blood urea nitrogen by week 4 . No marked difference of urine albumin concentrations between DES and NES groups were observed at any time points. These results suggested that pioglitazone might have contributed to the accelerated renal function recovery in diabetic subjects. The concentrations of plasma cytokines, including MCP-1, IL-6 and IFNy, showed no significant difference between DES and NES groups over the course of 8 weeks (Figure 8 ).

\section{Discussion}

Electrospun scaffolds have been extensively studied to acquire the capability of delivering therapeutic molecules to local tissues for an improved tissue regeneration.[24, 25] Illuminated by previous research, here we functionalized our electrospun scaffold to deliver pioglitazone to local islets in the hope of further enhancing the therapeutic effectiveness by reining in the hyperglycemia and the loss of renal functions. Previous research discovered that pioglitazone could prevent the apoptosis of islets, the onset of streptozotocin-induced diabetes and dysfunction of pancreatic $\beta$-cells.[26-28] SEM micrographs showed no marked difference of fiber morphology between DES and NES scaffolds, evidencing that the incorporation of pioglitazone would not introduce structural changes to the scaffold. This lack of structural change ensured that the enhanced functional output of transplanted islets derived from the pharmaceutical capability of pioglitazone. The in vitro kinetic study showed that the DES readily released pioglitazone into the milieu within a 4-day window. This rapid and sustained release would subject the transplanted islets to a sufficient yet temporary stimulation, which could minimize unwanted side effects. Meanwhile, the in vivo analysis showed that pioglitazone remained in the blood circulation at week 4 but got cleared by week 8 . This further attested the temporary nature of this pioglitazone therapy scheme. Moreover, the MTS result evidenced that islets retained their viability on DES scaffolds. Nor did the DES scaffold triggered massive apoptosis. These results together pointed out that DES scaffold was a biocompatible delivery vehicle for the rapid yet temporary release of pioglitazone to local islets.

The bioluminescent measurement demonstrated a steady proliferation of transplanted islets in both DES and NES groups. The fact that the islet population in the DES group outgrew its peer in the NES group, starting from week 4 , suggested that the stimulation by pioglitazone might have contributed to this proliferative advantage. It should be noted that the strength of the bioluminescent signal was positively associated with the mass of the islet population.[19] The immuno-staining also confirmed that transplanted islets in both groups contained insulin-secreting cells, confirming that transplanted islets were fully functional. To quantitatively compare the insulin secretion capability between DES and NES groups, we measured the blood concentrations of 
C-peptide 2 and insulin. Both C-peptide 2 and insulin secretions saw an increase in the DES group as early as week 4, which could be attributed to the accelerated proliferation of transplanted islets. Although it is a byproduct of insulin production with no known biological functions, C-peptide 2 serves as an important clinical indicator of functional output of islet transplantation because only endogenous insulin secretion would give rise to C-peptide production. The fact that both C-peptide and insulin levels in the DES group was higher than those in the NES group since week 4 suggested that the stimulation of pioglitazone boosted the insulin secreting capability of transplanted islets, possibly by targeting pancreatic $\beta$-cells in the islets because they are responsible for insulin production.[29] The increased insulin secretion in the DES group readily translated into a decreased blood glucose level, showing that an enhanced insulin secretion could alleviate the hyperglycemia in diabetic mice. Moreover, the mice in the DES group showed a stronger capability to withstand sudden glucose challenge, giving evidence to another benefit of the pioglitazone stimulation. These results demonstrated that the delivery of pioglitazone by an electrospun scaffold to local islets would be a promising means to manage the blood glucose level in diabetic patients following the islet transplantation.

Renal functions are frequently compromised or lost due to diabetes, necessitating a kidney transplantation. Since kidney transplantation is not readily
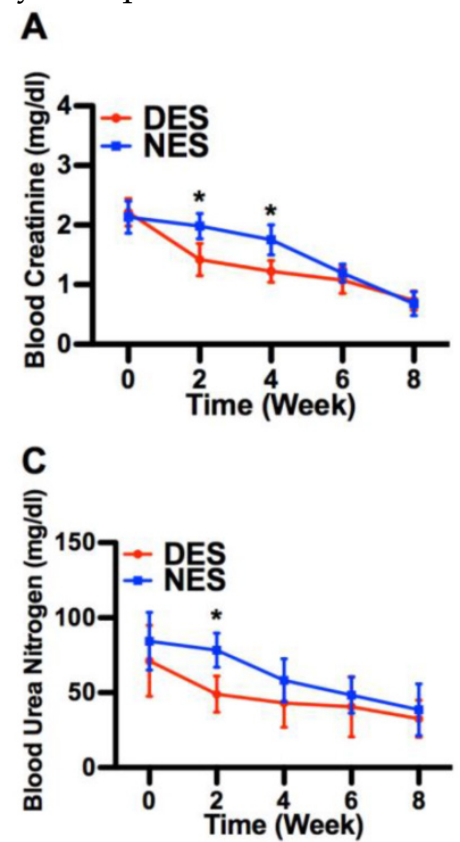

available, a strategy allowing the recovery of renal functions is of great significance. Our results demonstrated that the recovery of renal functions could be accelerated due to the pioglitazone stimulation. The levels of blood creatinine, urine creatinine, blood urea nitrogen and urine albumin all witnessed a drop over the 8-week window, suggesting an improved renal functions following the islet transplantation. Particularly, blood and urine creatinine in the DES group demonstrated an accelerated decrease on week 2 and 4, evidencing the therapeutic effect of the pioglitazone stimulation. These results suggested that the pioglitazone stimulation causes a synergistic effect in the treatment of diabetes, reigning in both the hyperglycemia and nephrotic disorders in diabetic subjects. In diabetic patients, the administration of pioglitazone frequently causes renal edema, a common culprit for nephrotic disorder, and mandates a cease of treatment. Guan et al demonstrated that pioglitazone could cause renal edema due to an enhanced renal $\mathrm{Na}^{+}$absorption via an ENaC mediated pathway.[30] This discovery suggested that pioglitazone might have the ability to impact renal tissues directly and argued for a revamped scheme for pioglitazone-based treatments for clinical benefits. Our results demonstrated that a reduce dosage of pioglitazone delivered by a DES scaffold could relieve moderate renal injury. This would offer an easy to implement engineering solution for a clinical challenge.

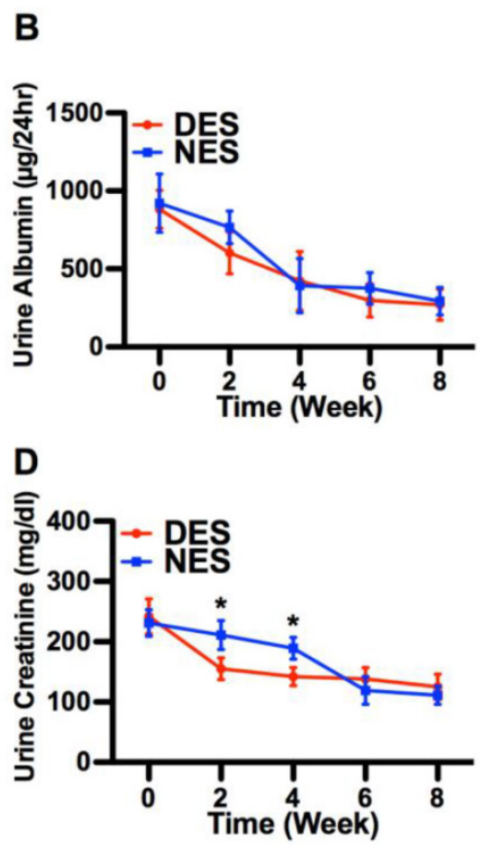

Figure 7. The recovery of renal functions. (A) blood creatinine; (B) urine albumin; (C) blood urea nitrogen; (D) urine creatinine. The creatinine concentrations in both blood and urine from the DES group were lower than those from the NES group on week 2 and 4 . In addition, a difference was observed in blood urea nitrogen on week 2 between DES and NES groups. This accelerated recovery of blood creatinine, urine creatinine and blood urea nitrogen evidenced the therapeutic effect of pioglitazone on renal functions. A star sign indicates a statistical difference between the groups ( $n=8$ in the DES group; $n=7$ in the NES group). 
A

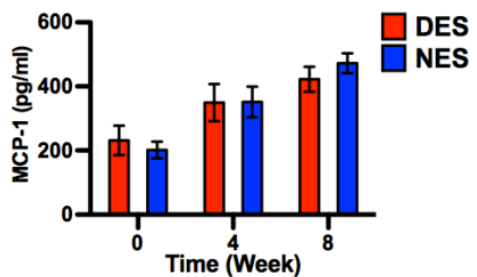

B

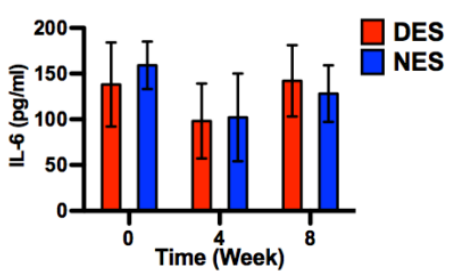

C

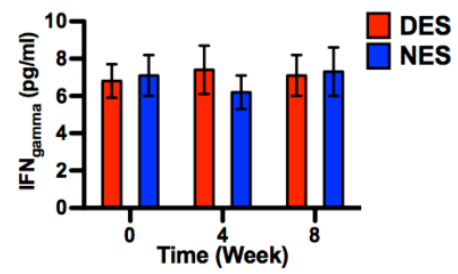

Figure 8. Plasma cytokine concentrations. (A) MCP-I; (B) IL-6; (C) IFNY. No significant difference was observed between DES and NES groups ( $n=8$ in DES group; $n=7$ in NES group).

\section{Conclusion}

The stimulation of transplanted islets to achieve a blood glucose hemostasis in diabetic patients has so far witnessed limited clinical success. In our previous studies, we successfully boosted the insulin secretion of transplanted islets with an electrospun scaffold, which could be attributed to a more favorable microenvironment, to manage blood glucose in diabetic mice. In light of recent discovery on therapeutic roles of pioglitazone on diabetes, in this study we functionalized the scaffold to be able to locally deliver pioglitazone to transplanted islets. The local stimulation of transplanted islets by pioglitazone resulted in an accelerated islet growth, an increased insulin secretion and consequently a lower blood glucose level in diabetic mice. Moreover, the alleviation of hyperglycemia also contributed to the recovery of renal functions compromised by the diabetes. These findings shed new lights on the bioengineering of therapeutic devices for diabetic patients in the future.

\section{Acknowledgement}

The authors declare no conflict of interests. This work was supported by National Natural Science Foundation of China (Grant Number: 30300357), Chongqing Application and Development Fund (Grant number: cstc2013yykfA10008), China's Ministry of Health Fund (W2013ZT150) and Shanghai Committee of Science and Technology Fund (13ZR1450400).

\section{Competing Interests}

The authors have declared that no competing interest exists.

\section{References}

1. Dor Y, Brown J, Martinez OI, Melton DA. Adult pancreatic beta-cells are formed by self-duplication rather than stem-cell differentiation. Nature. 2004; 429: 41-6.

2. Lee HC, Bonner-Weir S, Weir GC, Leahy JL. Compensatory adaption to partial pancreatectomy in the rat. Endocrinology. 1989; 124: 1571-5.

3. Donath MY, Storling J, Berchtold LA, Billestrup N, Mandrup-Poulsen T. Cytokines and beta-cell biology: from concept to clinical translation. Endocrine reviews. 2008; 29: 334-50.

4. Sorenson RL, Brelje TC. Adaptation of islets of Langerhans to pregnancy: beta-cell growth, enhanced insulin secretion and the role of lactogenic hormones. Hormone and metabolic research $=$ Hormon- und Stoffwechselforschung $=$ Hormones et metabolisme. 1997; 29: 301-7.

5. Meier JJ, Bhushan A, Butler AE, Rizza RA, Butler PC. Sustained beta cell apoptosis in patients with long-standing type 1 diabetes: indirect evidence for islet regeneration? Diabetologia. 2005; 48: 2221-8.

6. Zhao YF, Feng DD, Chen C. Contribution of adipocyte-derived factors to beta-cell dysfunction in diabetes. The international journal of biochemistry \& cell biology. 2006; 38: 804-19.

7. Karges B, Durinovic-Bello I, Heinze E, Boehm BO, Debatin KM, Karges W. Complete long-term recovery of beta-cell function in autoimmune type 1 diabetes after insulin treatment. Diabetes care. 2004; 27: 1207-8.

8. Rankin MM, Kushner JA. Adaptive beta-cell proliferation is severely restricted with advanced age. Diabetes. 2009; 58: 1365-72.

9. Tschen SI, Dhawan S, Gurlo T, Bhushan A. Age-dependent decline in beta-cell proliferation restricts the capacity of beta-cell regeneration in mice. Diabetes. 2009; 58: 1312-20.

10. Zhang X, Xu Y, Thomas V, Bellis SL, Vohra YK. Engineering an antiplatelet adhesion layer on an electrospun scaffold using porcine endothelial progenitor cells. Journal of biomedical materials research Part A. 2011; 97: 145-51.

11. Zhang $\mathrm{X}$, Thomas V, Xu Y, Bellis SL, Vohra YK. An in vitro regenerated functional human endothelium on a nanofibrous electrospun scaffold. Biomaterials. 2010; 31: 4376-81.

12. Zhang X, Thomas V, Vohra YK. Two ply tubular scaffolds comprised of proteins/poliglecaprone/polycaprolactone fibers. Journal of materials science Materials in medicine. 2010; 21: 541-9.

13. Thomas V, Zhang X, Vohra YK. A biomimetic tubular scaffold with spatially designed nanofibers of protein/PDS bio-blends. Biotechnology and bioengineering. 2009; 104: 1025-33.

14. Zhang $X$, Thomas V, Vohra YK. In vitro biodegradation of designed tubular scaffolds of electrospun protein/polyglyconate blend fibers. Journal of biomedical materials research Part B, Applied biomaterials. 2009; 89: 135-47.

15. Thomas V, Zhang X, Catledge SA, Vohra YK. Functionally graded electrospun scaffolds with tunable mechanical properties for vascular tissue regeneration. Biomed Mater. 2007; 2: 224-32.

16. Yin $\mathrm{H}$, Gao M, Leoni L, Han $\mathrm{H}$, Zhang $\mathrm{X}, \mathrm{Fu} \mathrm{Z}$. The therapeutic role of monocyte chemoattractant protein-1 in a renal tissue engineering strategy for diabetic patients. PloS one. 2013; 8: e57635.

17. Mooradian AD, Chehade J, Thurman JE. The role of thiazolidinediones in the treatment of patients with type 2 diabetes mellitus. Treatments in endocrinology. 2002; 1: 13-20.

18. Yin H, Park SY, Wang XJ, Misawa R, Grossman EJ, Tao J, et al. Enhancing pancreatic Beta-cell regeneration in vivo with pioglitazone and alogliptin. PloS one. 2013; 8: e65777.

19. Park SY, Wang X, Chen Z, Powers AC, Magnuson MA, Head WS, et al. Optical imaging of pancreatic beta cells in living mice expressing a mouse insulin I promoter-firefly luciferase transgene. Genesis. 2005; 43: 80-6.

20. Guo Z, Chong AS, Shen J, Foster P, Sankary HN, McChesney L, et al. Prolongation of rat islet allograft survival by the immunosuppressive agent leflunomide. Transplantation. 1997; 63: 711-6.

21. Yin D, Tao J, Lee DD, Shen J, Hara M, Lopez J, et al. Recovery of islet beta-cell function in streptozotocin- induced diabetic mice: an indirect role for the spleen. Diabetes. 2006; 55: 3256-63.

22. Zhong WZ, Williams MG. Simultaneous quantitation of pioglitazone and its metabolites in human serum by liquid chromatography and solid phase extraction. Journal of pharmaceutical and biomedical analysis. 1996; 14: 465-73.

23. Yamashita K, Murakami H, Okuda T, Motohashi M. High-performance liquid chromatographic determination of pioglitazone and its metabolites in human serum and urine. Journal of chromatography B, Biomedical applications. 1996; 677: 141-6.

24. Meinel AJ, Germershaus O, Luhmann T, Merkle HP, Meinel L. Electrospun matrices for localized drug delivery: current technologies and selected biomedical applications. European journal of pharmaceutics and biopharmaceutics : official journal of Arbeitsgemeinschaft fur Pharmazeutische Verfahrenstechnik eV. 2012; 81: 1-13.

25. Sill TJ, von Recum HA. Electrospinning: applications in drug delivery and tissue engineering. Biomaterials. 2008; 29: 1989-2006. 
26. Takamura T, Ando H, Nagai Y, Yamashita H, Nohara E, Kobayashi $K$. Pioglitazone prevents mice from multiple low-dose streptozotocin-induced insulitis and diabetes. Diabetes research and clinical practice. 1999; 44: 107-14.

27. Moritoh Y, Takeuchi K, Asakawa T, Kataoka O, Odaka H. Combining a dipeptidyl peptidase-4 inhibitor, alogliptin, with pioglitazone improves glycaemic control, lipid profiles and beta-cell function in $\mathrm{db} / \mathrm{db}$ mice. British journal of pharmacology. 2009; 157: 415-26.

28. Kawashima S, Matsuoka TA, Kaneto H, Tochino Y, Kato K, Yamamoto K, et al. Effect of alogliptin, pioglitazone and glargine on pancreatic beta-cells in diabetic $\mathrm{db} / \mathrm{db}$ mice. Biochemical and biophysical research communications. 2011; 404: 534-40.

29. Scharfmann R, Rachdi L, Ravassard P. Concise review: in search of unlimited sources of functional human pancreatic beta cells. Stem cells translational medicine. 2013; 2: 61-7.

30. Guan Y, Hao C, Cha DR, Rao R, Lu W, Kohan DE, et al. Thiazolidinediones expand body fluid volume through PPARgamma stimulation of ENaC-mediated renal salt absorption. Nature medicine. 2005; 11: 861-6. 